\title{
Análise Espacial Espectral Bidimensional em Partículas de Material Compósito $\mathrm{Al} / \mathrm{SiC}$
}

\author{
E.S.B. ARAÚJO ${ }^{1 *}$, L.S. BATISTA ${ }^{1}$ e J.D. SCALON ${ }^{2}$ \\ Recebido em 28 junho, 2013 / Aceito em 31 março, 2014
}

\begin{abstract}
RESUMO. Este trabalho investiga o padrão da distribuição espacial de partículas de carboneto de silício em uma liga de alumínio $(\mathrm{Al} / \mathrm{SiC})$ utilizando análise espacial espectral bidimensional. Esta técnica se baseia na transformada de Fourier da função de autocovariância amostral. A análise espectral é uma ferramenta poderosa para investigar o padrão espacial de pontos, uma vez que não assume características estruturais dos dados antes da análise e utiliza-se apenas da função espectral. As coordenadas dos centros das partículas foram adquiridas através de tecnicas de processamento de imagem. $\mathrm{O}$ espectro polar do periodograma e métodos de Monte-Carlo são usados para testar a hipótese de completa aleatoriedade espacial das partículas. Os resultados mostram que as partículas de carboneto de silício apresentam distribuição espacial completamente aleatória na liga de alumínio e que a análise espacial espectral pode ser uma ferramenta alternativa para investigar padrões de configurações pontuais no espaço.
\end{abstract}

Palavras-chave: espectro polar, processos pontuais, propriedade de materiais, distribuição espacial.

\section{INTRODUÇÃO}

Apesar das idéias da análise espectral serem difundidas e aplicadas em várias áreas do conhecimento (e.g. processamento de sinais), o potencial das mesmas para análise de configurações espaciais de eventos ainda é pouco explorado [4]. Bartlett [1] foi o pioneiro em propor o uso da análise espectral em processos pontuais, enquanto Renshaw \& Ford [6] foram os primeiros a mostrarem como a análise espectral pode ser uma importante ferramenta para a análise de configurações espaciais bidimensionais. A análise espectral tem sido usada em poucas áreas de conhecimento para análise de configurações espaciais no plano [4] e, salvo melhor juízo, ainda não foi utilizada na análise da distribuição espacial de partículas em materiais compósitos.

Analisar processos pontuais espaciais tem lugar de destaque em diversas áreas do conhecimento [3]. Por exemplo, a distribuicão espacial de segunda fase (partículas) é um dos fatores mais

\footnotetext{
*Autor correspondente: Edmary Silveira Barreto Araújo

${ }^{1}$ Instituto Federal da Bahia - IFBA, Departamento de Matemática, 40110-150 Salvador, BA, Brasil.

E-mails: ebarreto@ifba.edu.br; lurimar@ifba.edu.br

${ }^{2}$ Universidade Federal de Lavras - UFLA, Departamento de Ciências Exatas - DEX, 37200-000 Lavras, MG, Brasil. E-mail: scalon@dex.ufla.br
} 
importantes das propriedades mecânicas de materiais compósitos. Por exemplo, a tenacidade a fratura deste material depende não só da forma e da fracão de volume, mas também sobre a distribuição espacial do reforço de partículas na matriz e, portanto, a caracterização de tais distribuições é de primordial importância na ciência dos materiais [2]. A maior parte dos métodos disponíveis para a análise de processos pontuais no espaço é baseada em técnicas focadas no "Domínio Espacial" o que envolve a análise da distância entre pontos [3] e, portanto, são essas técnicas que, em geral, são utilizadas na análise da distribuição espacial de partículas em materiais compósitos [7].

No estudo de processos pontuais, cada evento pode ser idealizado como um ponto e a distribuição dos eventos em uma região como uma configuração espacial de pontos no plano. A componenente estocástica primária é a própria localização espacial do evento e, assim, o dado de interesse é a própria localização espacial (coordenada) de cada um dos eventos na área de estudo. Um dos principais objetivos da análise é estudar a distribuição espacial destes pontos, testando hipótese de completa aleatoriedade espacial do padrão observado contra as hipóteses alternativas de distribuições que apresentam eventos aglomerados ou regularmente distribuídos [3].

Neste artigo a análise espectral bidimensional é utilizada para caracterizar a distribuicão espacial das partículas de carboneto de silício localizadas em uma liga de alumínio, tendo em vista a importância dessa caracterização para avaliar as propriedades mecânicas desses materiais. A análise é conduzida a partir das coordenadas dos centros das partículas de carboneto de silício, extraídas através de técnicas de processamento de imagem, de uma amostra metalográfica de um material compósito (Al-SiC), produzida pelo Departamento de Engenharia de Materiais, da Universidade de Sheffield, Reino Unido. O espectro bidimensional para o padrão espacial é estimado pelo periodograma que é apresentado como o espectro de potências da transformada de Fourier. Os testes contra a hipótese de completa aleatoriedade espacial das partículas são realizados usando o espectro polar (funções $R$-espectro e $\theta$-espectro) e o método de Monte-Carlo.

\section{FUNÇÃO ESPECTRAL BIDIMENSIONAL}

Suponha que um padrão observado consiste de $N_{x}$ eventos em uma região retangular de lados $l_{1} \mathrm{e}$ que $l_{2}$ e $\left\{\left(x_{1 j}, x_{2 j}\right), j=1, \ldots, N_{x}\right\}$ denota a localização dos eventos. $\mathrm{O}$ espectro bidimensional para cada padrão é estimado pelo periodograma que esta baseado na transformada discreta de Fourier (TDF) das coordenadas $\left\{x_{j}\right\}[4]$.

$$
F(p, q)=\sum_{j=1}^{N_{x}} e^{-2 \pi i\left(\frac{p x_{1 j}}{l_{1}}+\frac{q x_{2 j}}{l_{2}}\right)}=A(p, q)+i B(p, q)
$$

onde $p=0, \pm 1, \pm 2, \ldots$ e $q=0, \pm 1, \pm 2, \ldots$.

O periodograma, também chamado de função espectral amostral, pode ser calculado como o espectro de potências da transformada discreta de Fourier das coordenadas

$$
\hat{f}\left(w_{p}, w_{q}\right)=|F(p, q)|^{2}=\{A(p, q)\}^{2}+\{B(p, q)\}^{2},
$$


em que $\left(w_{p}, w_{q}\right)=\left(\frac{2 \pi p}{l_{1}}, \frac{2 \pi q}{l_{2}}\right)$.

Uma ferramenta que a análise espectral apresenta é avaliar o padrão em meio a escala e direção. Em [4], os autores utilizam o periodograma em coordenadas polares para definirem dois resumos unidimensionais do periodograma. Cada $\hat{f}\left(w_{p}, w_{q}\right)$ pode ser representado na forma polar usando a notação $\hat{g}\left(w_{r}, w_{\theta}\right)$, em que $r=\sqrt{p^{2}+q^{2}}$ e $\theta=\tan ^{-1}\left(\frac{p}{q}\right)$.

Os valores médios das ordenadas do periodograma, para valores similares de $r$, investigam a escalas de padrão sob a hipótese de isotropia. O $R$-espectro, $\hat{f}_{R}(r)$, é definido como

$$
\hat{f}_{R}(r)=\frac{1}{n_{r}} \sum_{r^{\prime}} \sum_{\theta} \hat{g}\left(w_{r^{\prime}}, w_{\theta}\right), \quad r=1,2, \ldots,
$$

em que a soma total é dividida por $n_{r}$ ordenadas do periodograma e $r-1<r^{\prime} \leq r$.

Os valores médios das ordenadas do periodograma, para valores similares de $\theta$, investigam características direcionais de padrão. O $\theta$-espectro, $\hat{f}_{\theta}(\theta)$, é definido como

$$
\hat{f_{\theta}}(\theta)=\frac{1}{n_{\theta}} \sum_{r} \sum_{\theta^{\prime}} \hat{g}\left(w_{r}, w_{\theta^{\prime}}\right), \quad \theta=0^{0}, 10^{0}, 20^{0}, \ldots, 170^{0},
$$

em que a soma total é dividida por $n_{\theta}$ coordenadas do periodograma para os quais $\theta-5^{0}<\theta^{\prime} \leq$ $\theta+5^{0}$.

A análise espectral também pode ser aplicada a uma rede bidimensional de dados. As posições aproximadas de eventos de um padrão espacial pontual podem ser representadas pela interseção de uma malha fina sobreposta na área de estudo. Este método foi proposto pelos autores em [6] e descrito a seguir.

A autocovariância amostral é definida como

$$
C_{j k}=\frac{1}{m n} \sum_{s=1}^{m-j} \sum_{\Omega_{t}} X_{s t} X_{s+j, t+k}
$$

em que $\Omega_{t}=\{1, \ldots, n-k ; k \geq 0\}, \Omega_{t}=\{-k+1, \ldots, n ; k<0\}$ e $X_{s t}(s=1, \ldots, m$; $t=1, \ldots, n)$ é a matriz bidimensional de observações corrigida para a média.

A matriz de autocorrelação é dada por $\left\{\frac{C_{j k}}{s^{2}}\right\}$, em que $s^{2}$ é a variância da amostra $\left\{X_{s t}\right\}$.

A matriz completa de autocorrelação espacial correlaciona os dados perfeitamente ente si, ou seja, tem um valor central de $\frac{C_{00}}{s 2}=1$, em que $C_{00}$ está definida na Equação 2.4.

$$
C_{00}=\frac{1}{m n} \sum_{s=1}^{m} \sum_{t=1}^{n} X_{s t}^{2}
$$

Uma descrição mais compacta do padrão espacial em processos pontuais é obtida pelo periodograma ou função espectral amostral. 
O periodograma é calculado via a autocovariância amostral vista na Equação 2.3 e, portanto, o periodograma fica definido por

$$
\hat{f}\left(w_{p}, w_{q}\right)=\sum_{j=-m+1}^{m-1} \sum_{k=-n+1}^{n-1} C_{j k} \cos \left(j w_{p}+k w_{q}\right) .
$$

Substituindo na Equação 2.5 o valor da frequência $\left(w_{p}, w_{q}\right)=\left(\frac{2 \pi p}{m}, \frac{2 \pi q}{n}\right)$, tem-se

$$
P_{p q} \equiv \hat{f}\left(\frac{2 \pi p}{m}, \frac{2 \pi q}{n}\right)=m \cdot n\left(a_{p q}^{2}+b_{p q}^{2}\right),
$$

em que $a_{p q}$ é a parte real e $b_{p q}$ é a parte imaginária da Equação 2.7 definida como

$$
a_{p q}+i b_{p q}=\frac{1}{m n} \sum_{s=1}^{m} \sum_{t=1}^{n} X_{s t} e^{2 \pi i\left(\frac{p s}{m}+\frac{q t}{n}\right)} .
$$

Desenvolvendo a Equação 2.7, pode-se encontrar os valores

$$
a_{p q}=\left(\frac{1}{m . n}\right) \sum_{s=1}^{m} \sum_{t=1}^{n} X_{s t} \cos \left[2 \pi\left(\frac{p s}{m}+\frac{q t}{n}\right)\right]
$$

e

$$
b_{p q}=\left(\frac{1}{m . n}\right) \sum_{s=1}^{m} \sum_{t=1}^{n} X_{s t} \operatorname{sen}\left[2 \pi\left(\frac{p s}{m}+\frac{q t}{n}\right)\right]
$$

A ampla gama de frequência é sobre $p=0, \ldots, m-1 ; q=0, \ldots, n-1$ mas, devido a relação de simetria, considerar-se somente parte do periodograma, ou seja,

$$
p=0, \ldots, \frac{m}{2} ; q=-\frac{n}{2}, \ldots, \frac{n}{2}-1 \text {. }
$$

\section{INTERVALO DE CONFIANÇA}

Para contrução do intervalo de confiança, baseado no método de Monte Carlo, foram gerados valores de $R$-espectro e $\theta$-espectro a partir das Equações 2.1 e 2.2, respectivamente.

A seguir, são gerados $n$ configurações sobre a hipótese de completa aleatoriedade espacial e para cada uma dessas configurações calculam-se o $R$-espectro e $\theta$-espectro. Em seguida, ordena-se os $n$ valores $R$-espectros para cada escala do menor para o maior e também, ordena-se os n valores $\theta$-espectro para cada ângulo. São também gerados os valores de $R$-espectro e $\theta$-espectro para as configurações observadas.

Tem-se o limite inferior do intervalo obtido pelo $i$-ésimo $n$-quantil e o limite superior pelo $(n-i)$ ésimo $n$-quantil com $\left(1-\frac{i}{100}\right)$ de confiança.

Todos as análises foram realizadas utilizando programas desenvolvidos no software $\mathrm{R}$ [5]. O software livre R é muito utilizado para análise estatistica e simulação por profissionais da área. 


\section{MATERIAL COMPÓSITO AL/SIC}

A amostra metalográfica de um material compósito (Al/SiC), carboneto de silício na liga de alumínio, foi produzida pelo Departamento de Engenharia de Materiais, Universidade de Sheffield, Inglaterra. As partículas de segunda fase de carboneto de silício tinham uma fração de volume igual a $11 \%$. A área da amostra metalográfica foi aproximadamente $192 \times 288 \mu \mathrm{m}$. A amostra metalógrafa foi colocada em um analisador de fase óptico do microscópio controlado por computador, o que permitiu o ajuste totalmente automático, a focagem, o posicionamento e a digitalização da amostra. A ampliação do microscópio total utilizada foi de 600 vezes, obtendose um tamanho de pixel de $0,375 \mu \mathrm{m}$. Assim, uma imagem digital foi gerada com área igual a $512 \times 767$ pixels quadrados. A imagem digital bidimensional foi analisada usando técnicas de processamento de imagem para extrair as coordenadas (centros) de cada partícula dentro da imagem. A imagem real foi de $512 \times 767$ pixels, dos quais usamos apenas as partículas localizadas em $512 \times 512$ pixels, produzindo 419 partículas em amostra $S_{1}$. Os $512 \times 512$ imagens foram transformadas em uma área quadrada com uma unidade para facilitar a análise [7]. Os dados da Figura 1 representam as posições das particulas do material compósito $\mathrm{Al} / \mathrm{SiC}$ na matriz de aluminio.

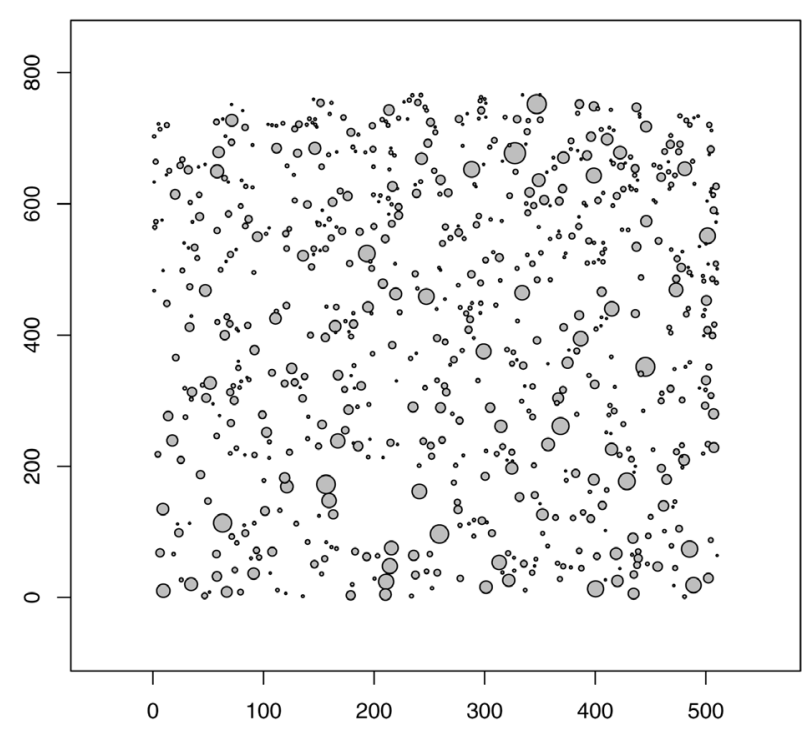

Figura 1: Imagem $(512 \times 767)$ pixels da distribuição espacial das 730 partículas de reforço na liga de alumínio.

\section{ANÁLISE ESPACIAL ESPECTRAL BIDIMENSIONAL}

Uma ferramenta que pode ser útil para a uma primeira análise exploratória da distribuição espacial das partículas é o diagrama de pontos formado pelos centróides das partículas. Os mapas de pontos da amostra apresentados nas Figuras 2(a) e 2(b) fornecem uma ideia da distribuição espacial das partículas de $\mathrm{SiC}$ ao longo da matriz de alumínio entretanto, analisando essas figuras 
não é possível avaliar claramente qual o padrão espacial da distribuição das partículas, ou seja, aleatório, agrupado ou regular.

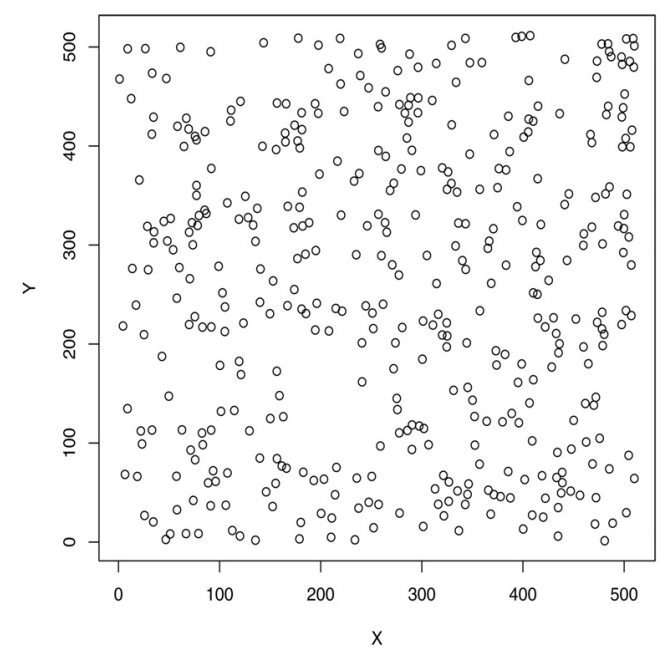

(a) Configuração pontual no quadrado de $512 \times 512$ pixels.

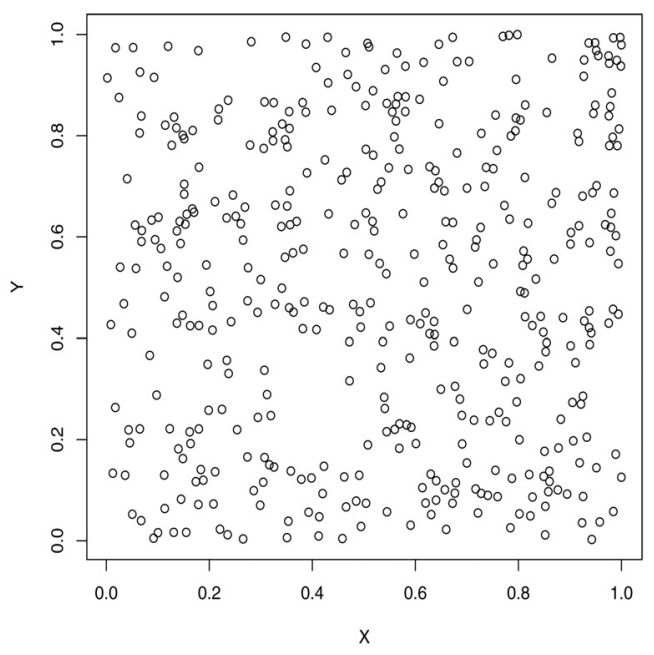

(b) Configuração pontual no quadrado da unidade.

Figura 2: A Distribuição espacial das coordenadas de 419 partículas de reforço da amostra.

Para uma análise mais rigorosa do padrão da distribuição espacial das partículas é necessário utilizar métodos estatísticos mais avançados, como aqueles baseados em distâncias entre eventos [3] ou análise espectral [4]. Neste trabalho realiza-se a análise das partículas de carboneto de silício na liga de alumínio usando a teoria da análise espectral bidimensional.

Os dados do periodograma apresentados na Figura 3, calculados utilizando a Equação 2.6, representam porções da variância da amostra em diferentes frequências. A Figura 3 mostra que as estimativas da variância estão próximas de zero nas diferentes frequências, de tal modo que não observa-se uma frequência que domina a forma do periodograma. Assim, a forma do periodograma não apresenta grandes variações, refletindo a asusência de estrutura espacial na configuração observada. As flutuações observadas nos valores do periodograma podem ser creditadas inteiramente a variações amostrais. Essas características observadas na Figura 3 fornecem indícios de que a distribuição das partículas de SiC é completamente aleatória na base de alumínio.

A função $R$-espectro apresentada na Figura 4(a) mostra escalas de padrão sob a hipótese de isotropia, enquanto a função $\theta$-espectro apresentada na Figura 4(b) mostra características direcionais da configuração espacial.

A Figura 4(a) e Figura 4(b) mostram que as funções $R$-espectro e $\theta$-espectro encontram-se dentro do envelope de completa aleatoriedade espacial com 0,99 de confiança e, portanto, aceitando a hipótese de que as partículas de carboneto de silício apresentam uma distribuição completamente aleatória na liga de alumínio. 


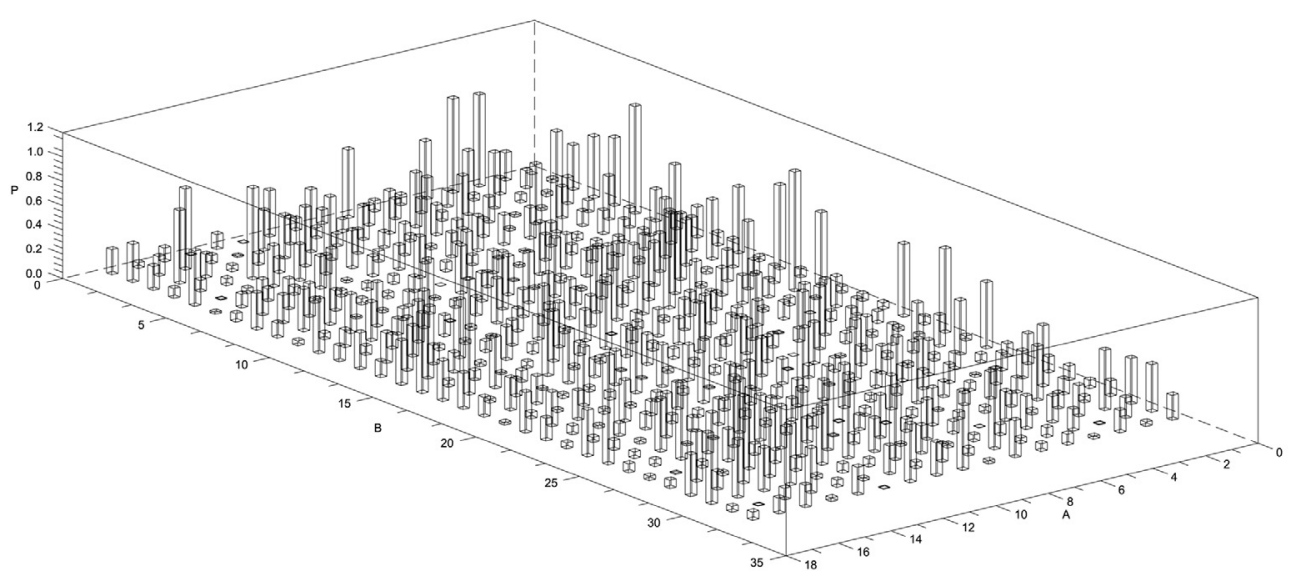

Figura 3: Periodograma da distribuição espacial das partículas de $\mathrm{SiC}$ na matriz de alumínio.

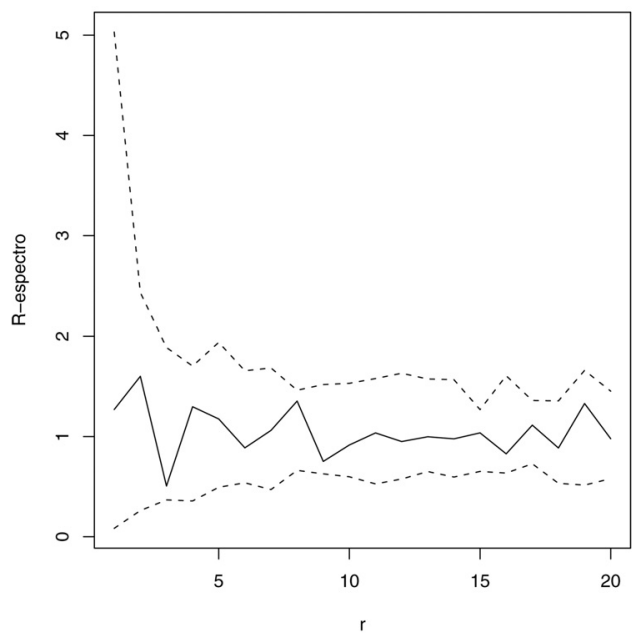

(a) $R$ espectro

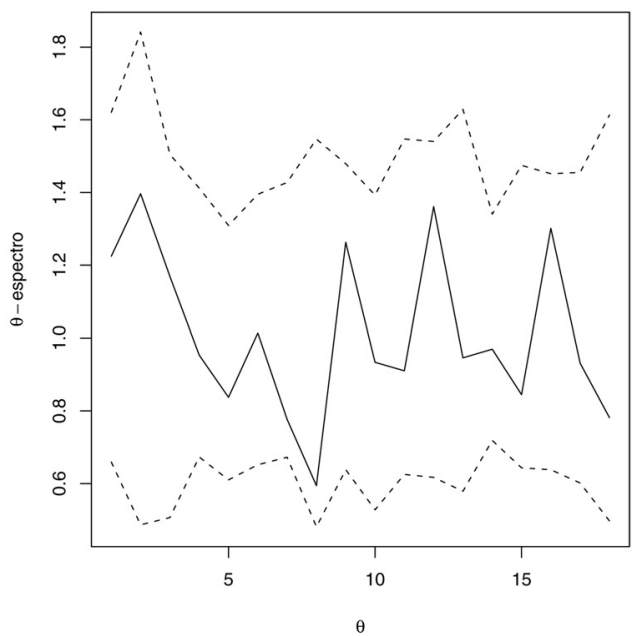

(b) $\theta$ espectro

Figura 4: Espectro polar observado da distribuição das partículas no material compósito $\mathrm{Al} / \mathrm{SiC}$ (linha contínua) juntamente com os intervalos de confiança (linhas tracejadas) construídos a partir de 99 simulações de Monte Carlo sob a hipótese de completa aleatoriedade espacial.

A distribuicão espacial de segunda fase está intimamente relacionada com as propriedades mecânicas observadas em materiais compósitos e, portanto, pode ser utilizada como um indicador da qualidade do material. Por exemplo, a tenacidade a fratura deste material depende não somente da forma e da fracão de volume, mas também da distribuição espacial do reforço de partículas na matriz [2]. Sabe-se que um material terá uma maior chance de apresentar fratura em locais com agrupamento de partículas [2]. Assim, investigar a distribuição espacial de partículas em materiais compósitos é de primordial importância na ciência dos materiais. 
O resultado obtido neste trabalho indica que o material compósito, representado pela amostra metalográfica analisada, apresenta uma qualidade desejável quanto a distribuição espacial aleatória das partículas (aleatoriedade). Entretanto, este resultado diverge ligeiramente do resultado obtido por Scalon et al. [7] que mostrou, utilizando análises baseadas em distâncias entre pontos, que as partículas dessa amostra metalográfica apresentam uma distribuição regular. Na verdade, Scalon et al. [7] afirmam que a pequena regularidade detectada no padrão espacial é devida aos tamanhos das partículas que não foram levados em consideração na análise baseada nos centros das partículas.

Seria prematuro tirar conclusões definitivas sobre o valor comparativo da técnica espectral com outras técnicas estatísticas disponíveis tendo como base apenas uma amostra. Entretanto, apesar da análise espectral não ser de fácil uso como as análises baseadas em distâncias entre eventos, a mesma se apresenta como uma ferramenta alternativa e poderosa para investigar padrões pontuais, uma vez que não assume qualquer suposição estrutural dos dados antes da análise, como a isotropia, que é difícil de ser observada em diversas situações práticas como observam alguns pesquisadores como [1], [3], [4] e [6].

\title{
6 CONCLUSÃO
}

A análise espacial espectral mostrou que as partículas de carboneto de silício apresentam distribuição espacial completamente aleatória na liga de alumínio. Os resutados também indicam que a análise espacial espectral pode ser ferramenta alternativa para investigar padrões de configurações pontuais no espaço.

\section{AGRADECIMENTOS}

Os autores agradecem a Helen Atkinson (University of Sheffield) pela amostra metalográfica utilizada no artigo e a CAPES pelo apoio financeiro.

\begin{abstract}
This work investigates the spatial distribution pattern of silicon carbide particles in an aluminum alloy $(\mathrm{Al} / \mathrm{SiC})$ using two-dimensional spectral spatial analysis. This technique is based on the Fourier transform of the autocovariance function sample. The spectral analysis is a powerful tool for investigating the spatial distribution of points, since it does not assume structural characteristics of the data before analysis and uses only the spectral function. The coordinates of the centers of the particles were acquired through image processing techniques. The polar periodogram spectrum and Monte-Carlo methods are used to test the hypothesis of complete spatial randomness of the particles. The results show both the silicon carbide particles have completely random spatial distribution in the aluminum alloy and the spatial spectral analysis can be an alternative tool to investigate point patterns in space.
\end{abstract}

Keywords: polar spectrum, point processes, material properties, spatial distribution. 


\section{REFERÊNCIAS}

[1] M.S. Bartlett. The spectral analysis of two-dimensional point processes. Biometrika, 51 (1964), 299-311.

[2] K.K. Chawla. "Composite Materials: Science and Engineering”. Springer, New York, (2012).

[3] P.J. Diggle. "Statistical Analysis of Spatial Point Patterns". Academic Press, London, (1983).

[4] M.A. Mugglestone \& E. Renshaw. A practical guide to the spectral analysis of spatial point processes. Computational Statistics and Data Analysis, 21 (1996), 43-65.

[5] R Development Core Team. "R: a language and environment for statistical computing". $R$ Foundation for Statistical Computing, Vienna, (2012).

[6] E. Renshaw \& D. Ford. The descriptions of spatial using two-dimensional spectral analysis. Vegetatio, 56 (1984), 75-85.

[7] J.D. Scalon, N.R.J. Fieller, E.C. Stillman \& H.V. Atkinson. Spatial pattern analysis of second-phase particles in composite materials. Materials Science and Engineering, A356 (2003), 245-257. 